\title{
COMPORTAMENTO EXCÊNTRICO DO EFEITO TUNING EM SÍSMICA 4D
}

\author{
Marco C. Schinelli' \\ Recebido em 2 de abr., 2002 / Aceito em 12 de nov., 2003 \\ Received Abr. 2, 2002 / Accepted Nov. 12, 2003
}

\section{ABSTRACT}

The feasibility analysis of seismic monitoring of fluids flow in reservoirs, through consecutive seismic acquisition (known as 4D or time-lapse seismic), should not involve just the the laboratorial evaluation of modifications on physical response caused by the fluids substitution with the consequent variation of the elastic parameters, but mainly a previous phase of seismic modeling, whose importance is among others, to quantify the impact of the tuning effect that can subvert the expected amplitude variations after the time-lapse. In this sense such effect needs to be carefully investigated because, due to fluids substitution at the reservoirs level, the tuning variation can introduce a very important factor of influence on the seismic amplitudes, what can makes unfeasible the interpretation of the seismic 4D.

Keywords: Time-lapse seismic, 4D, tuning.

\section{RESUMO}

A análise da viabilidade técnica para monitoramento do fluxo de fluidos em reservatórios, através de levantamentos sísmicos do tipo time-lapse, ou 4D, deve envolver não apenas a avaliação laboratorial das modificações provocadas no reservatório pela substituição de fluidos com a con seqüente variação dos parâmetros elásticos mas, principalmente, uma fase prévia de modelagem sísmica cuja importância pode ser, dentre outras, quantificar o imp acto do efeito tuning que pode afetar as esperadas variações de amplitude após o time-lapse. Nesse caso, tal efeito precisa ser investigado com profundidade, pois devido à substituição de fluídos ao nível dos reservatórios, a variação do tuning observado em camadas delgadas pode introduzir um fator-modificação das amplitudes sísmicas que inviabilize a correta interpretação da sísmica 4D, especialmente quando se procura fazer uma interpretação mais quantitativa com a análise do comportamento de AVO e outras técnicas que exigem a investigação da variação de amplitudes.

Palavras-chave: monitoramento sísmico; 4D; interferência na forma do pulso. 


\section{INTRODUÇÃOO}

Muitos são os requisitos técnicos necessários para assegurar 0 sucesso de uma aquisição sísmica do tipo time-lapse (4D), como é normalmente referenciada. Os elevados investimentos para aquisição de sucessivas imagens 3D sobre uma mesma área, necessárias à monitorização do movimento de fluidos no reservatório, tornam-se técnica bastante dispendiosa - 0 que por si só justifica quaisquer esforços para seu perfeito planejamento. Vários são os autores - Lumley, Behrens e Wang - que já empenharam esforços na identificação de instrumentos para quantificação da viabilidade técnica de levantamentos desse tipo. Dentre os vários fatores de risco para um levantamento $4 \mathrm{D}$ está a capacidade de discriminação temporal do reservatório a ser monitorado. Neste contexto, a resolução sísmica e a espessura do reservatório são elementos fundamentais a considerar.

Com base na análise de modelagen sísmica podem ser feitas predições sobre o efeito do tuninge a capacidade de monitoramento do 4D, e obtidas informações importantes para a estratégia de aquisição, processamento e interpretação dos resultados do time-lapse.

\section{ETAPA DE ANÁLISE DA VIABILIDADE TÉCNICA}

0 Campo em foco, cujo mapa estrutural sísmico ao nível do topo do reservatório, está mostrado na Figura l, foi escolhido para um estudo da viabilidade téenica de monitoramento da injeção de $\mathrm{CO}^{2}$, através do uso de sísmica 4D. Dentre as etapas dessa análise constou a estimativa da eficácia do monitoramento sísmico, usando técnica de ranqueamento segundo critérios sugeridos por técnicos da Chevron (ver bibliografia) e apresentada na Tabela 1. Observar que os valores da escala variam de 0 a 45 (valor máximo correspondente ao prospecto ideal). Nessa classificação, $04 \mathrm{D}$ analisado situou-se no grau 27, um valor pouco acima da média para prospectos 4D. Entretanto, a metodologia usada desconsidera a importância de fatores altamente críticos que podem até mesmo inviabilizar o sucesso do time-lapse

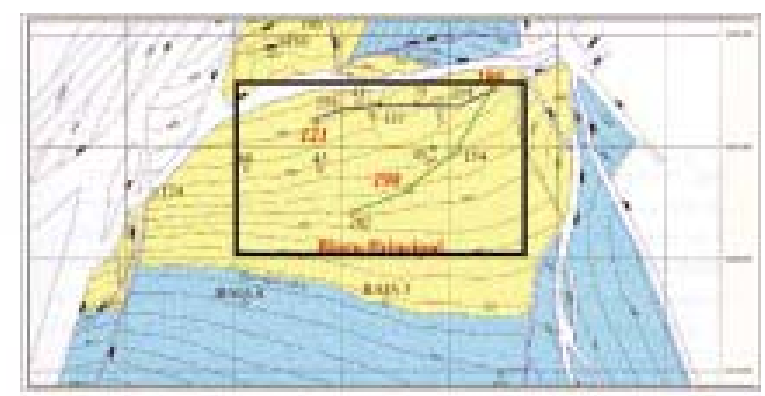

Figura 1 - Bloco principal do campo estudado Figure I - Localization of the studied area
Tabela 1 - Quantificação dos fatores de risco para o 4D proposto Table 1 - Quantification of the risk factors for the 4D proposed
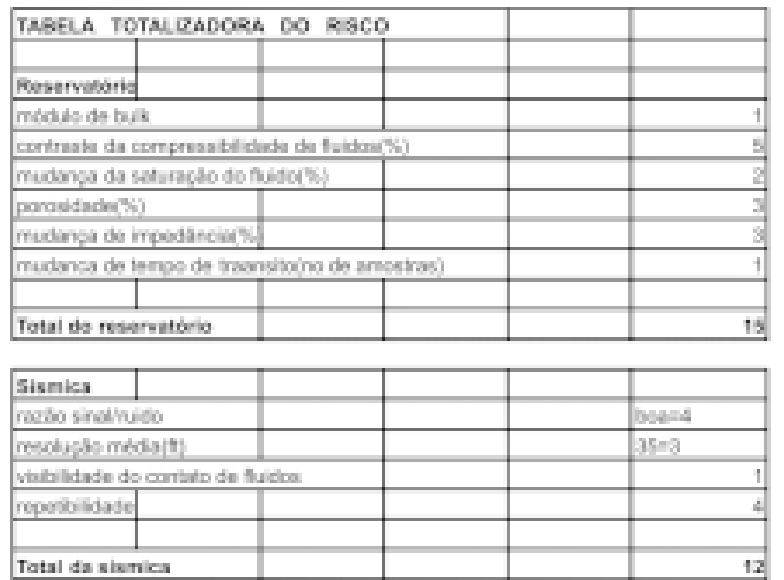

Escore Toto

\section{MODELAGEM DO EFEITO TUNING}

0 poço usado na análise, encontrava-se, na época da aquisiçã̃o dos perfis, na área do campo dentro da capa de gás para o intervalo do reservatório a ser estudado. Para efeito da modelagem foi usada a substituição inversa, isto é, adicionou-se óleo ao intervalo compreendido entre 0 topo da Fm. Sergi e o paleosolo subjacente - que funciona como selo limitador daquele intervalo em relação às outras zonas do reservatório. É também na porção superior desse intervalo que esta sendo injetado o $\mathrm{CO}^{2}$. Como para efeitos práticos o gás associado e $\mathrm{o} \mathrm{CO}^{2}$ a ser injetado têm diferenças de gravidade que pouco influenciam os resultados, foram usados os parâmetros do gás do reservatório.

A Figura 2 mostra 0 trecho analisado no poço. Observar que 0 intervalo tem espessura em torno de $15 \mathrm{~m}$. Atentar também para a presença da camada de paleosolo cujo aparecimento marca o limite inferior da primeira zona do reservatório Sergi na área. Este paleosolo, que tem expressão regional, é constituído de pacotes sílticos argilosos e tem espessura de até $3 \mathrm{~m}$. Seu comportamento sísmico é marcado por um alto valor de impedância, superior ao do arenito sobrejacente 0 que normalmente resulta num alto coeficiente de reflexão na interface, que devido a proximidade da reflexão do topo da zona superior do Sergi provoca forte interferência das duas reflexões. 0 objetivo da modelagem foi investigar o comportamento de amplitudes no topo do reservatório e principalmente a interferência provocada pelo paleosolo. 


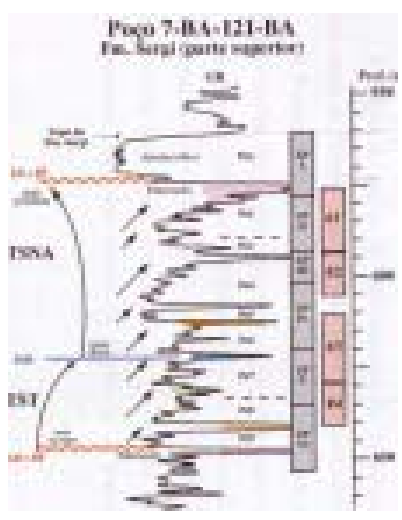

Figura 2 - 0 Paleo-solo- Tem expressão regional e espessura média de 3m, sendo constituído de pacotes síltico-argilosos com alto coeficiente de reflexão.

Posiciona-se entre 15 a 30m do topo da Fm. Sergi

Figure 2 - The hardground has regional expression and medium thickness of $3 \mathrm{~m}$, being constituted by shaly sandstones with high reflection coefficient. It is positioned among 15 to $30 \mathrm{~m}$ bellow the top of Sergi formation.

Para maior fidelidade durante a modelagem foi extraído do próprio dado sísmico o pulso a ser usado na modelagem. Nos casos em que não se tenha ainda adquirido 0 3D base, a análise poderia ser feita com um pulso extraído de uma linha $2 \mathrm{D}$ da área com parâmetros aproximados aos que se pretende usar no 4D. A importância dessa etapa é: trabalhar na modelagem com a resolução sísmica real que é um dos requisitos fundamentais para análise do efeito do tuning. A fase do pulso sísmico pode ser estimada por correlaçãa entre 0 sismograma sintético e a sísmica disponível.

Para avaliação da relaçãa $\mathrm{Vp} / \mathrm{Vs}_{\text {s no }}$ reservatório foi usada a fórmula empírica de Greenberg/Castangna .

Apesar das aproximações decorrentes, desde que se conheça a litologia e porosidade com certa precisão os valores são aceitáveis para uma estimativa dessa natureza.

\section{ANÁLISE DOS RESULTADOS}

A Tabela 2 mostra a variação de amplitude estimada ao nível do topo da Fm. Sergi, do poço modelado para diferentes saturaçōes em amplitude e impedância elástica. Nas situações extremas de saturaçãa observar que a variação da posição do paleosolo provoca reduções de até $20 \%$ nas amplitudes.

A Figura 3 mostra os perfis sintéticos, baseados nas propriedades médias reais dos intervalos e nas diferentes espessuras, entre os topos da Fm. Sergi e do paleosolo, usados na modelagem do Efeito Tuning, utilizando a waveletextraída dos dados sísmicos.
Tabela 2 - Valores de amplitude para diferentes saturações, medidos em amplitude convencional e impedância elástica. Os 3 valores em cada coluna correspondem à amostragem dos lobos superior, principal e inferior da waveletcorrespondente a reflexão no topo do reservatório.

Table 2 - Amplitude value for different saturations, measured in conventional amplitude and elastic impedance. The three values in each column correspond to the samples from the upper, middle and lower wavelet lobes corresponding to the reflection in the top of the reservoir

\begin{tabular}{|c|c|c|c|c|c|c|c|c|}
\hline Princtos & Dhroibide & VP & $\sqrt{5}$ & $\begin{array}{l}\text { Amp } \\
\text { sads }\end{array}$ & $\begin{array}{l}\text { Armp } \\
\text { fo }\end{array}$ & $\begin{array}{l}\text { Arpp. } \\
y r\end{array}$ & $\begin{array}{l}\text { Ant } \\
\text { af }\end{array}$ & $\begin{array}{l}\text { Vrisios } \\
\text { s }\end{array}$ \\
\hline Finbotpo & 2.14 & 2565 & 124 & 411 & 4) 4 & .90 & -312 & 0 \\
\hline Boind: & 244 & 3722 & 2118 & 611 & SII & 69 & 346 & 25 \\
\hline $\cos -10 \%$ & & & & -452 & की। & -45 & -125 & 15 \\
\hline Anfinde & 221 & 144 & 120 & -353 & 44 & $.5 \pi$ & -149 & \\
\hline \multirow[t]{2}{*}{ 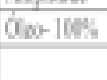 } & 299 & 3445 & 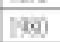 & 611 & 783 & $6 \%$ & 367 & \\
\hline & & & & $-44)$ & 499 & 364 & -363 & \\
\hline & & & & & & & & \\
\hline In dix & & & & .1232 & 4654 & . 1351 & t5I & 115 \\
\hline \multirow[t]{3}{*}{$\cos -10 \%$} & & & & 1451 & 1927 & 1900 & 835 & 37 \\
\hline & & & & 447 & 688 & -87 & -114 & 15 \\
\hline & & & & & & & & \\
\hline lm dix & & & & 597 & .1324 & .1015 & -253 & \\
\hline \multirow[t]{2}{*}{ (6ov- $16 \%$} & & & & 1767 & 2175 & {$[901$} & 1312 & \\
\hline & & & & +93 & 54 & $\cdot \pi 2$ & -324 & \\
\hline
\end{tabular}

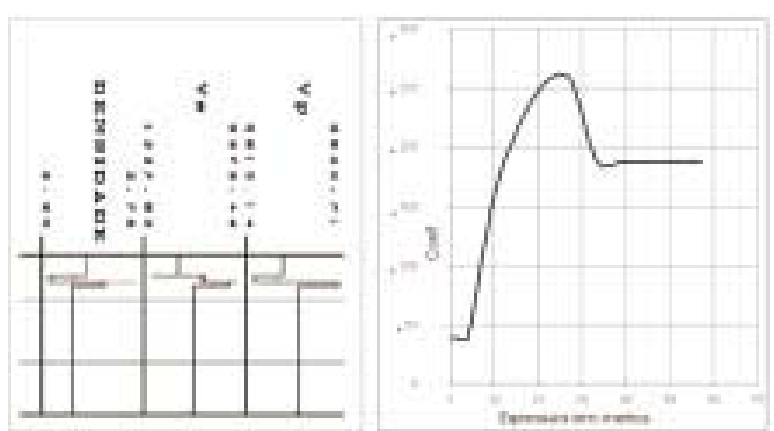

Figura 3 - Perfis modelados para situaçãa original, após substituição de fluidos (esquerda) e função funing correspondente para várias espessuras (direita).

Figure 3-Modeled logs for original situation and after fluids substitution (left) and the function corresponding to the tuning for different thickness (right).

Usando as resposta de amplitude modelada para os perfis sintético, com variadas espessuras, foram gerados mapas de amplitude, através de modelagem convolucional simples № mapa da Figura 4, tem-se a distribuição dos valores que seriam obtidos na interface 
correspondente ao topo da Fm. Sergi, com base na modelagem do tuning. Nesse mapa, os valores de amplitude já estão influenciados pelo tuning, com a condição de saturação original do reservatório e poderiam, numa

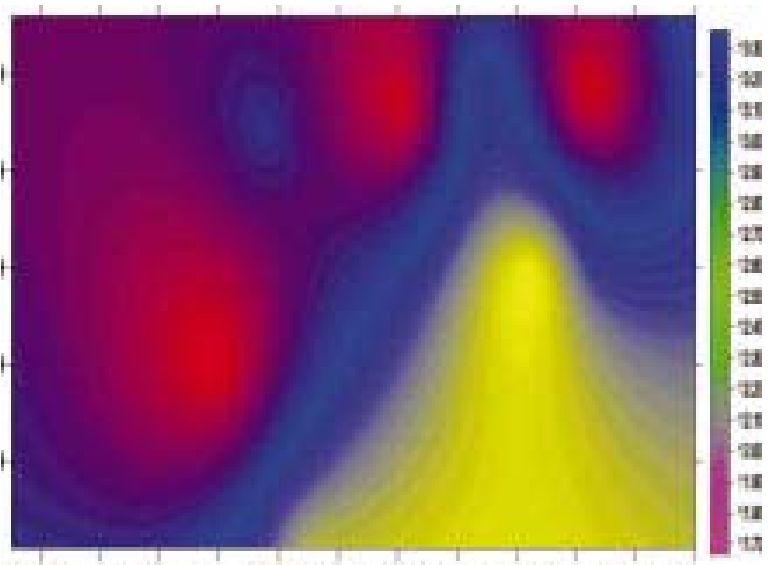

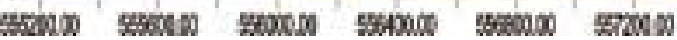

\begin{abstract}
Figura 4 - Mapa de amplitudes modeladas para a situação original de saturação do reservatório. Figure 4-Modeled amplitude map for the original reservoir saturation condition.
\end{abstract}

análise precipitada, ser correlacionados com a presença de hidrocarbonetos no intervalo. Nos mapas seguintes são simuladas as diferenças de amplitude que seriam observadas após a injeção, em duas situaçōes distintas: saturação total de óleo de 100\% para todos os poços (Figura 5) e 50\% apenas para os três poços mais a nordeste da

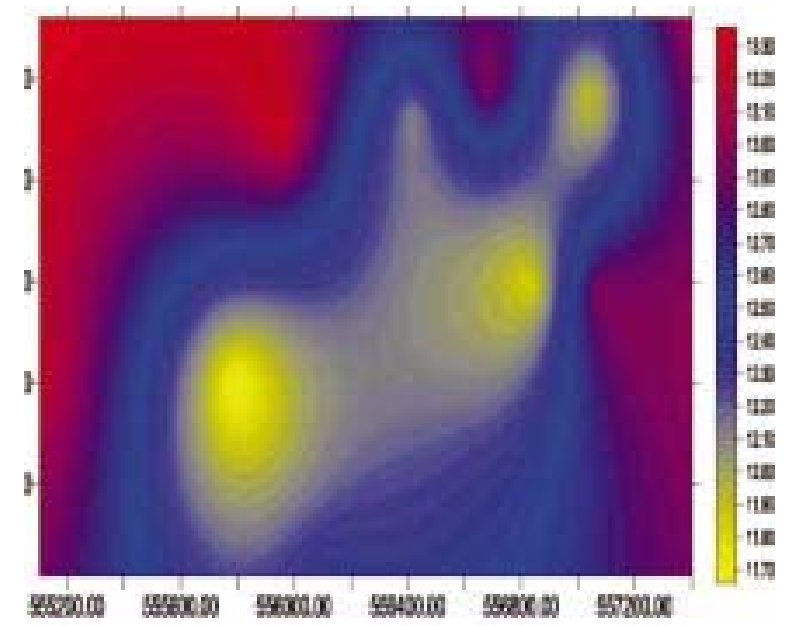

Figura 5 - Mapa de amplitudes para 100\% de óleo em todos os poços.

Figure 5 - Amplitude map for $100 \%$ of oil in all the wells área (Figura 6). Observa-se que os mapas são influenciados pela variaçã̃o do efeito de tuning e não refletem fielmente as variações de saturação consideradas - 0 que pode dificultar a interpretação dos resultados do time-lapse -, falseando a predição da anisotropia do fluxo e conseqüentemente influenciar negativamente a estratégia da injeção.

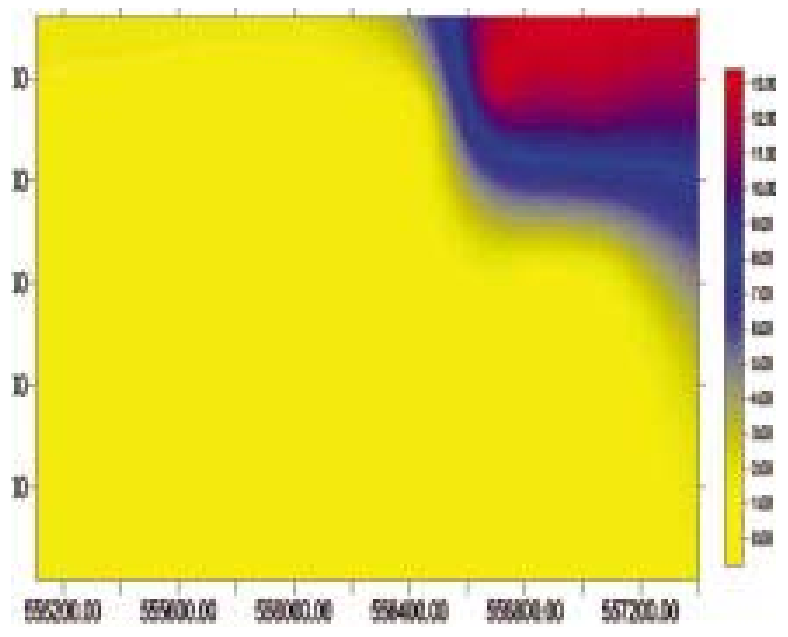

Figura 6 - Mapa de amplitudes para 50 \% de saturação de óleo apenas para três poços mais a NE da área.

Figure 6-Amplitude map for $50 \%$ of oil saturation

only on the wells on the northeast portion of the area.

A variação no efeito do tuning, após a substituição de fluidos, deve-se a variação dos atrasos na reflexão no topo do paleosolo, que é diferente para cada poço, ocasionada pela variação de saturação e conseqüentemente variação de $\mathrm{Vp}$.

\section{CONCLUSÕES E RECOMENDAÇÕES}

Fica evidente que o efeito tuning tem comportamento excêntrico em situações onde o reservatório passe por substituição de fluidos e tal variação do efeito pode até mesmo impedir a interpretação da sísmica 4D ou correlação fiel dos mapas de diferenças de amplitudes com a variação de saturação ao nível dos reservatórios. Assim sendo, é importante, especialmente para reservatórios delgados, onde a variação de tempo de trânsito devido às modificações de velocidade de propagação no reservatório são imperceptíveis, restando apenas a variação de amplitudes como ferramenta de interpretação do 4D, que se avalie profundamente 0 efeito tuning. Esse tipo de análise oferece ainda informações adicionais que podem ajudar nas etapas de aquisição, processamento (por exemplo, recomendando diminuição do intervalo 
de amostragem, maior atenção na recuperação de altas freqüências através de algorítimos de deconvolução, inversão para IE etc) e mesmo na interpretação onde o uso de atributos "não-tradicionais" - freqüência instantânea que é boa indicadora de efeitos de tuning; análise do comportamento de AVO e outras técnicas que podem ajudar na estimativa e remoção da influência do efeito de tuning na resposta do $4 D$-, e finalmente permitir correlacionar (de forma mais confiável) variações de amplitudes com diferenças de saturação.

\section{Agradecimentos}

À Petrobrás pela oportunidade de apresentação deste trabalho e aos colegas Aurino Aragão, Benildo Casanova, Alcides Aggio e Eduardo Ferrer pela contribuição para diversas etapas desta análise .

\section{REFERÊNCIAS}

LUMLEY, David E;. BEHRENS, Ronald; WANG, Zhiijing. Assesing the technical risk of a 4-D seismic project. The Leading Edge, La Habra, v. 16, n. 9, p. 1287-1291, September 1997. Chevron Petroleum Technology Company, 1998.

SAVINI, R.; OLIVEIRA, Flávio M. de. Análise faciológica e estratigráfica da Porção Superior da Formação Sergi no bloco principal do campo estudado na Bacia do Reconôncavo. Petrobrás, E\&P-BA/GEXP,GELAB. Relatório interno, ago. 1994.

LIN, T. L.; PHAIR, R. Texaco Exploration \& Production, Inc., AVO Tuning. In: ANNUAL MEETING INTERNATIONAL, 63., 1993, [S. I.]. Expanded Abstracts. [S. I.: S. n.], 1993, p. 727-730.

\section{NOTA SOBRE O AUTOR}

Marco Cesar Schinelli é graduado em Geologia pela Universidade Federal da Bahia em 1979, trabalha desde então na Petrobras, já tendo atuado nas áreas de aquisição, processamentos sísmico terrestre e maríimo, interpretação explotatória e exploratória. Atualmente, como consultor sênior, se dedica a processos de caracterização sísmica de reservatórios em campos terrestres maduros da bacia do Recôncavo, com particular interesse em sísmica multicomponente, 4D, sísmica de poço e técnicas avançadas de interpretação sísmica. 